\title{
EVEREST II randomized clinical trial: Predictors of mitral valve replacement in de novo surgery or after the MitraClip procedure
}

\author{
Donald Glower, MD, ${ }^{\mathrm{a}}$ Gorav Ailawadi, MD, ${ }^{\mathrm{b}}$ Michael Argenziano, MD, ${ }^{\mathrm{c}}$ Michael Mack, MD, ${ }^{\mathrm{d}}$ \\ Alfredo Trento, MD, ${ }^{\mathrm{e}}$ Andrew Wang, MD, ${ }^{\mathrm{a}} \mathrm{D}$. Scott Lim, MD, ${ }^{\mathrm{b}}$ William Gray, MD, ${ }^{\mathrm{c}}$ Paul Grayburn, MD, ${ }^{\mathrm{d}}$ \\ John Dent, MD, ${ }^{\mathrm{b}}$ Linda Gillam, MD, ${ }^{\mathrm{c}}$ Barathi Sethuraman, MD, ${ }^{\mathrm{f}}$ Ted Feldman, MD, ${ }^{\mathrm{g}}$ Elyse Foster, MD, \\ Laura Mauri, MD, ${ }^{\mathrm{i}}$ and Irving Kron, $\mathrm{MD},{ }^{\mathrm{b}}$ on behalf of the EVEREST II Investigators
}

\begin{abstract}
Objective: The Endovascular Valve Edge-to-Edge Repair Study (EVEREST II) is a prospective, multicenter, randomized controlled trial comparing percutaneous repair with the MitraClip device to mitral valve (MV) surgery in the treatment of mitral regurgitation. The present study analyzed the patient characteristics and treatment effects on mitral repair versus replacement.
\end{abstract}

\begin{abstract}
Methods: Of 279 patients enrolled, 80 surgical patients underwent 82 MV operations and 178 underwent an initial MitraClip procedure, of whom 37 underwent a subsequent MV operation within 1 year of their index the MitraClip procedure. A logistic regression model was used to predict MV replacement according to valve pathology, etiology of mitral regurgitation, age, previous cardiac surgery, and treatment group.
\end{abstract}

Results: The rate of percutaneous or surgical MV repair at 1 year was $89 \%(158 / 178)$ in patients initially receiving the MitraClip device versus $84 \%(67 / 80)$ in the surgical patients $(P=.36)$. Surgical repair was performed after the MitraClip procedure in $20(54 \%)$ of 37patients $(P<.001$ vs surgery). In both the MitraClip device and surgery groups, MV replacement was significantly associated with anterior leaflet pathology $(P=$ .035). Logistic regression analysis showed that anterior leaflet pathology predicted MV replacement. In 5 $(13.5 \%)$ of 37 patients undergoing surgery after MitraClip therapy, replacement was performed in part because of MV injury associated with the MitraClip procedure.

Conclusions: These data suggest that anterior leaflet pathology is strongly associated with MV replacement in patients undergoing either de novo MV surgery or surgery after MitraClip therapy. MitraClip therapy has a repair rate similar to surgery through 1 year but also imparts a risk of replacement of a potentially repairable valve. (J Thorac Cardiovasc Surg 2012;143:S60-3)

In patients undergoing surgery for mitral regurgitation (MR), mitral repair has advantages over mitral replacement of improved durability, less anticoagulation, and better preservation of left ventricular function. ${ }^{1}$ As mitral repair techniques have improved, some investigators have concluded that nearly all regurgitant mitral valves (MVs) with

From the Duke University Medical Center, ${ }^{a}$ Durham, NC; University of Virginia Health System, ${ }^{\mathrm{b}}$ Charlottesville, Va; Columbia University Medical Center, ${ }^{\mathrm{c}}$ New York, NY; Baylor University Medical Center, ${ }^{\mathrm{d}}$ Dallas, Tex; Cedars-Sinai Medical Center, ${ }^{\mathrm{e}}$ Los Angeles, Calif; Abbott Vascular, ${ }^{\mathrm{f}}$ Menlo Park, Calif; Evanston Hospital, ${ }^{\mathrm{g}}$ NorthShore University Health System, Evanston, Ill; University of California, ${ }^{\text {h }}$ San Francisco, San Francisco, Calif; and Brigham and Women's Hospital, ${ }^{\mathrm{i}}$ Harvard Clinical Research Institute, Harvard Medical School, Boston, Mass.

Disclosures: Donald Glower, Gorav Ailawadi, Michael Argenziano, Michael Mack, Alfredo Trento, Andrew Wang, D. Scott Lim, William Gray, Paul Grayburn, John Dent, Linda Gillam, Barathi Sethuraman, Ted Feldman, Elyse Foster, Laura Mauri, and Irving Kron have nothing to disclose with regard to commercial support.

Presented at The American Association for Thoracic Surgery Mitral Conclave, New York, New York, May 5-6, 2011.

Received for publication May 15, 2011; revisions received Dec 28, 2011; accepted for publication Jan 16, 2012.

Address for reprints: Donald Glower, MD, Duke University Medical Center, Box 3851, Durham, NC 27710 (E-mail: Glowe001@mc.duke.edu).

$0022-5223 / \$ 36.00$

Copyright (c) 2012 by The American Association for Thoracic Surgery doi: $10.1016 /$ j.jtcvs.2012.01.047 degenerative disease can and should be repaired. ${ }^{2}$ However, large population-based samples have shown MV repair rates of only $50 \%{ }^{3}$ Factors proposed to predict MV replacement instead of repair include surgeon factors, ${ }^{3,4}$ older patient age, ${ }^{4}$ anterior or bileaflet mitral pathology, ${ }^{4}$ previous mediastinal radiotherapy, ${ }^{5}$ calcification, ${ }^{4}$ previous cardiac surgery, and functional etiology of MR. ${ }^{6}$ Most of these studies are potentially subject to patient selection bias and problems from retrospective data analysis.

The Endovascular Valve Edge-to-Edge Repair Study (EVEREST II) is a prospective, multicenter, randomized controlled trial comparing the MitraClip system with MV surgery in the treatment of MR. ${ }^{7,8}$ Data from the EVEREST II trial were analyzed to meet 4 objectives:

1. To determine whether the overall repair rate is comparable between patients who underwent the MitraClip procedure versus de novo surgery.

2. To determine the specific baseline demographic or valve characteristics that are predictive of MV replacement.

3. To assess the MitraClip procedure effect on the ability to surgically repair the valve.

4. To evaluate the effect of surgeon experience on MV replacement. 


\section{Abbreviations and Acronyms}

EVEREST II = Endovascular Valve Edge-to-Edge Repair Study

MV = mitral valve

MR $=$ mitral regurgitation

\section{METHODS}

After individual institutional review board approval, 279 patients were enrolled at 37 sites in North America and randomized 2:1 (184 to the MitraClip device and 95 to surgery) in the EVEREST II randomized controlled trial. ${ }^{9}$ Patients with functional MR or degenerative MR involving anterior, posterior, or bileaflet disease were eligible for enrollment, and repair and replacement surgery were considered acceptable outcomes of the trial in the surgery group. Before randomization, the surgeons were asked to indicate whether surgical MV repair versus replacement was planned.

A comparison of the overall repair rate between the patients who underwent the MitraClip procedure versus de novo surgery was performed using Fisher's exact test.

Specific baseline demographic or valve characteristics were assessed for association with MV repair versus replacement on univariate analyses and in a multivariate logistic regression model, including the following variables: anterior or bileaflet leaflet pathology, functional etiology of MR, previous cardiac surgery, patient age, and treatment group (MitraClip vs surgery). Three MitraClip patients who were not implanted with a MitraClip device and did not undergo subsequent MV surgery were excluded from the present analysis because they received no treatment (repair or replacement) for MR.

MV injury or difficulty removing the MitraClip device was assessed by retrospectively reviewing the operative notes. To assess surgeon experience, surgeons were asked to provide the number of MV repairs and replacements performed in the year before enrolling patients in the EVEREST II randomized controlled trial.

The baseline characteristics between 2 groups were compared using Fisher's exact test for categorical variables and Student $t$ test for continuous variables.

\section{RESULTS}

\section{Surgical Results Through 1 Year in MitraClip and Surgery Groups}

Of the 279 patients, 178 were randomized and treated in the MitraClip group, and 80 were randomized to the surgery group. The planned surgical repair rate before randomization was $92 \%$ for treated patients in both the MitraClip and surgery groups. Through 1 year, $37(21 \%)$ of $178 \mathrm{Mi}-$ traClip patients underwent subsequent MV surgery, of whom $20(54 \%)$ underwent MV repair. The planned repair rate for the 37 patients who underwent surgery after the MitraClip procedure $(92 \%)$ was significantly greater than the actual repair rate $(54 \%, P=.005)$. The number of MitraClip devices implanted (17 with no clip, 7 with 1 clip, and 13 with 2 clips) was not associated with MV replacement $(P=.12)$. The median interval to MV surgery after the MitraClip procedure for these 37 patients was 41 days, with $28(76 \%)$ of 37 performed within 90 days from the index procedure. As previously reported by
Feldman and colleagues, ${ }^{7}$ the reason for surgery in these 37 patients was no MitraClip device implanted in 17, MR grade $3+$ or $4+$ after MitraClip implantation before hospital discharge in 5 , MR grade $3+$ or $4+$ after single leaflet device attachment in 9, and MR grade $3+$ or $4+$ after MitraClip implantation after hospital discharge despite dual leaflet attachment in 3 and symptoms in 3.

In the de novo surgery group, 67 (84\%) of 80 patients underwent MV repair surgery $(P<.001$ vs surgery after MitraClip) through 1 year. In this group, the planned surgical repair rate was $92 \%(P=.14,84 \%$ [67/80] vs $92 \%$ [60/67]). Two surgery patients underwent MV replacement as a second operation after the initial repair (Figure 1).

\section{Overall Repair (Percutaneous and Surgical) and Replacement Rates Through 1 Year}

Among the 178 patients who underwent the MitraClip procedure in the MitraClip group, 158 (89\%) underwent repair, either percutaneous $(n=138)$ or surgery $(n=20)$ after the MitraClip procedure, and was not significantly different from the repair rate in the surgery group (67/80 [84\%], $P=.36)$ through 1 year.

\section{Predictors of MV Repair Versus Replacement}

The baseline characteristics of the MitraClip $(n=178)$ and surgery $(\mathrm{n}=80)$ groups, including age, functional etiology of MR, previous cardiac surgery, and anterior anterior/bileaflet pathology were not significantly different $(P=.47)$. When all patients were combined (percutaneous and surgical), the only variable that showed a trend toward a difference between groups was anterior/bileaflet pathology on univariate analysis, with the patients undergoing replacement showing a trend toward a greater incidence of anterior/bileaflet pathology ( $47 \%$ vs $28 \%, P=.055)$.

Age, functional etiology of MR, previous cardiac surgery, incidence of anterior/bileaflet pathology), and treatment group (MitraClip device vs surgery) were included in a logistic regression model to determine whether any of these variables was associated with MV replacement. Anterior/bileaflet pathology was the only variable significantly associated with MV replacement $(P=.037)$. In the present small sample size, the association between MitraClip treatment and MV replacement was not significant $(P=.086)$.

\section{Effect of MitraClip Procedure on Ability to Surgically Repair the Valve}

Of the 37 patients who underwent surgery after the MitraClip procedure, 15 (41\%) underwent replacement when repair was planned before randomization, 19 (51\%) underwent repair as planned preprocedurally, $2(5 \%)$ underwent replacement as planned preprocedurally, and the remaining patient $(3 \%)$ underwent MV repair when replacement was planned $(n=1)$. 


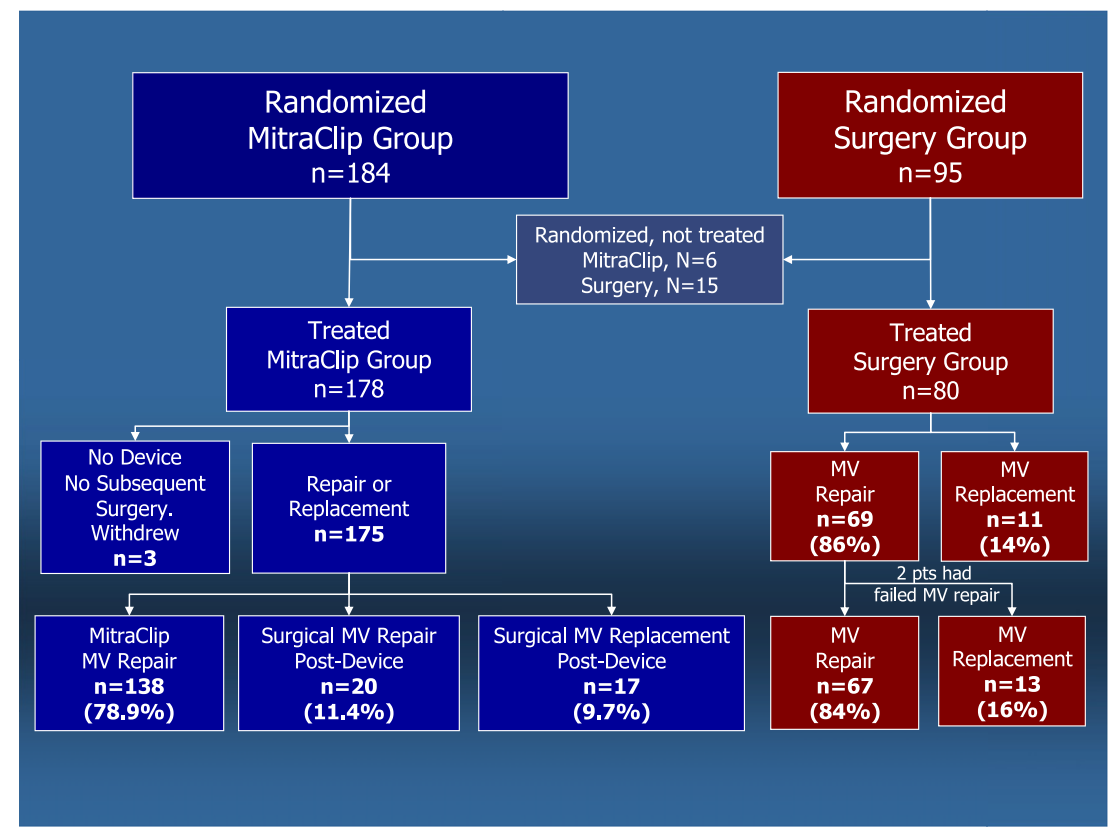

FIGURE 1. Type of procedure (percutaneous or surgical) performed through 1 year for MitraClip and surgery groups.

Of the 37 cases of MV surgery after the MitraClip procedure, valve injury was reported by the surgeon in $11(30 \%)$; 6 cases were reported to be related to the MitraClip procedure and the remaining 5 were reported to be related to difficulty in removing the device during explantation. In 6 of the 11 cases in which MV injury was reported, MV repair was able to be performed as planned; however, in the remaining 5 cases, the surgeon chose to replace the valve $(5 / 37[13.5 \%])$. Of these 5 patients, 4 had previously been implanted with 2 MitraClip devices, and the remaining patient was not implanted with a MitraClip device. Replacement was performed 6, 33, 41, 52, and 54 days after the MitraClip procedure. The surgical repair rate in patients noted to have MV injury was not different from the remaining patients undergoing surgery after the MitraClip device (6/11 [54\%] vs $15 / 26$ [58\%], $P=.89)$. To compare the effect of the interval to surgery after the MitraClip procedure on the surgical mitral repair rate, the repair rate of MitraClip patients undergoing surgery after 90 days (4/10 [40\%]) was compared with the repair rate in patients undergoing surgery within 90 days $(13 / 27$ [48\%], $P=.95)$.

Of the 80 patients in the surgery group, $8(10 \%)$ underwent replacement when repair was planned preprocedurally, $67(84 \%)$ underwent repair as planned preprocedurally, and $5(6 \%)$ underwent replacement as planned preprocedurally. For those 8 de novo surgery patients who underwent unplanned replacement, the reasons for the replacement were recurrent MR requiring repeat operation in 2, attempted repair but residual MR present intraprocedurally in 4, advanced age in 1, and bileaflet disease with myxomatous leaflets in 1.

\section{Effect of Surgeon Experience on MV Replacement}

In centers in which patients underwent MV surgery after the MitraClip procedure $(\mathrm{n}=37)$, the surgeon had performed a mean of $62 \mathrm{MV}$ surgeries (40 repairs and 22 replacements; median, 52 in the year before participation in EVEREST II). An average of $60 \mathrm{MV}$ surgeries (38 repairs and 22 replacements; median, 52) were performed in centers in which MitraClip patients underwent MV repair $(\mathrm{n}=20)$. An average of $64 \mathrm{MV}$ surgeries (41 repairs and 23 replacements; median, 52) were performed in the centers in which 17 MitraClip patients underwent MV replacement.

In the centers for the surgery group, the mean number of MV surgeries performed in the year before EVEREST II was 60 (39 repairs and 21 replacements; median, 45) in the 67 de novo surgery patients who underwent MV repair. It was 61 (39 repairs and 22 replacements; median, 46) in the 13 de novo surgery patients who underwent MV replacement. On examining the de novo surgery and surgery after the MitraClip patients combined, no difference was found in surgeon experience $(P=.87)$ when all patients who underwent MV repair $(\mathrm{n}=87)$ were compared with those patients who underwent MV replacement $(\mathrm{n}=30)$.

\section{CONCLUSIONS}

The EVEREST II trail provides unique data for patients with surgical MR owing to prospective data collection and central echocardiographic core laboratory analysis. The EVEREST II data confirm previously reported that anterior and bileaflet prolapse are associated with replacement versus repair. Patient age, functional etiology of MR, previous cardiac surgery, surgeon experience, and interval from 
MitraClip procedure could not be confirmed as risk factors for replacement in the present series. These data also show that the 1-year mitral repair rate after initial MitraClip treatment was similar to that of de novo surgery $(158 / 178$ [89\%] vs $67 / 80[84 \%])$.

However, the multivariate analysis and intraoperative findings suggest some increased likelihood of ultimate MV replacement associated with MitraClip therapy. The lower surgical repair rate after MitraClip placement versus de novo surgery (20/37 [54\%] vs 67/80 [84\%]) resulted in part from valves failing after the MitraClip procedure had a greater prevalence of anterior/bileaflet pathology. However, in $5(14 \%)$ of the 37 patients undergoing surgery after MitraClip, replacement occurred after MV injury associated with the MitraClip procedure or device explantation.

Although the likelihood of undergoing MV replacement instead of repair because of MitraClip therapy was not high $(5 / 178[3 \%])$, some caution is warranted in patient selection for MitraClip therapy. Furthermore, because all patients with unsuccessful initial repairs (either by MitraClip or surgery) did not ultimately return for surgery, it is impossible to know how many more unplanned replacements might have occurred in this cohort if a subsequent operation were performed. Rogers and colleagues ${ }^{9}$ reported successful MV repair up to 5 years after MitraClip placement, and the 5 patients requiring unanticipated mitral replacement after MitraClip therapy all underwent surgery fewer than 60 days after MitraClip placement.

Surgical extraction of the MitraClip from a mitral leaflet can be as simple as lifting the MitraClip device from the leaflets early after MitraClip placement. However, after 30 days after MitraClip placement, the MitraClip device will have some degree of surrounding fibrosis that might need surgical debridement, along with opening the arms of the MitraClip device to facilitate explantation. ${ }^{10}$ Explanting the MitraClip device from the leaflets is facilitated by pulling the central lock harness of the MitraClip, thereby opening the grippers and arms of the MitraClip device..$^{10} \mathrm{In}$ creased surgical experience with MitraClip removal techniques will likely improve the ease of mitral repair after MitraClip placement.
The EVEREST II data offer important insight into patient selection for MitraClip therapy. The potential for MitraClip therapy to reduce the likelihood of surgical repair would be of little concern to patients who have no surgical options or who have a greater risk of surgical mortality or who have limited life expectancy. Patients who are surgical candidates with a high probability of surgical repair will have to weigh the safety advantages of percutaneous MitraClip therapy against the possibility of ultimately needing mitral replacement instead of repair because of previous MitraClip placement.

In conclusion, using these data from the EVEREST II trial, selected patients who have high operative risk of surgical mitral repair might be the most appropriate candidates for MitraClip therapy at this early stage in the development of this novel technology. Additional follow-up will elucidate the long-term effects of MitraClip placement on mitral reparability.

\section{References}

1. Ogutu P, Ahmed I, Dunning J. Should patients with asymptomatic severe mitral regurgitation with good left ventricular function undergo surgical repair? Interact Cardiovasc Thorac Surg. 2010;10:299-305.

2. Lawrie GM, Earle EA, Earle N. Intermediate-term results of a nonresectional dynamic repair technique in 662 patients with mitral valve prolapse and mitral regurgitation. J Thorac Cardiovasc Surg. 2011;141:368-76.

3. Bolling SF, O'Brien SM, Brennan J, Li S, Prager RL, Gammie JS. Predictors of mitral valve repair: clinical and surgeon factors. Ann Thorac Surg. 2010;90: 1904-12.

4. Gillinov AM, Blackstone EH, Nowicki ER, et al. Valve repair versus valve replacement for degenerative mitral valve disease. J Thorac Cardiovasc Surg. 2008;135:885-93.

5. Crestanello JA, McGregor CGA, Danielson GK, et al. Mitral and tricuspid valve repair in patients with previous mediastinal radiation therapy. Ann Thorac Surg. 2004;78:826-31

6. Gillinov AM, Wierup PN, Blackstone EH, et al. Is repair preferable to replacement for ischemic mitral regurgitation? J Thorac Cardiovasc Surg. 2001;122: $1125-41$.

7. Feldman T, Foster E, Glower DD, et al. Percutaneous repair or surgery for mitral regurgitation. N Engl J Med. 2011;364:1395-406.

8. Mauri L, Garg P, Massaro JM, et al. The EVEREST II trial: design and rationale for a randomized study of the Evalve MitraClip system compared with mitral valve surgery for mitral regurgitation. Am Heart J. 2010;160:23-9.

9. Rogers JH, Yeo KK, Carroll JD, et al. Late surgical mitral valve repair after percutaneous repair with the MitraClip system. J Card Surg. 2009;24: 677-81.

10. Ladich E, Michaels MB, Jones RM, et al. Pathological healing response of explanted MitraClip devices. Circulation. 2011;123:1418-27. 